\title{
Integrated Native-Ethernet Optical Ring Networks for Scalable Customer-Owned Application
}

\author{
Satoru Fujimoto Non-member (Graduate School of Engineering, \\ Osaka Prefecture University, satorufj@eis.osakafu-u.ac.jp) \\ Taiji Sakamoto Non-member (Graduate School of Engineering, \\ Osaka Prefecture University, taiji@eis.osakafu-u.ac.jp) \\ Osanori Koyama Non-member (Graduate School of Engineering, \\ Osaka Prefecture University, koyama@eis.osakafu-u.ac.jp) \\ Yutaka Katsuyama Member (Graduate School of Engineering, \\ Osaka Prefecture University, katsu@eis.osakafu-u.ac.jp)
}

Keywords: Optical MAN, IP-over-WDM, optical ring network, enterprise network

We have proposed and examined One-by-One (O-O) and point-to-point $(\mathrm{P} 2 \mathrm{P})$ link integration scheme over nativeEthernet optical ring networks for scalable customer-owned MAN application. The network configurations are described to integrate the 2 optical links by using CWDM technologies. The 2-link integration enables us to construct economical optical ring networks, which was clarified by smaller number of optical transceivers (OTRs) required to provide IP services. This is the essential econimical point, because the transmssion equipment costs are main, after the optical fibers are owned in the CO-MAN.

An experimental network was constructed on the nativeEthernet base and the performance was examined to clarify the integrated CO-MAN characteristics. An optical coupler and a splitter to mux/demux 9 wavelengths of CWDM standard were fabricated for use in an edge node, and $2 \lambda$ - and $1 \lambda$-ADMs were fabricated for use in user buildings. It was estimated from the measured losses and the dynamic range of 1000Base-LX transceivers used in the experiment that the optical fiber length of the ring could be longer than $36.5 \mathrm{~km}$ to integrate $8 \mathrm{P} 2 \mathrm{P}$ links and one $\mathrm{O}-\mathrm{O}$ link without optical amplification.

The packet routing functions were implemented in the layer-3 switches (L3SWs). The routing by $\mathrm{P} 2 \mathrm{P}$ link was based on OSPF and that by O-O link was based on static routing, including the packet bypassing function to the control ring, when a failure occurred in the service ring. The throughput measurement clarified that the integration of the $\mathrm{O}-\mathrm{O}$ and $\mathrm{P} 2 \mathrm{P}$ links was successfully made for the ring network connecting one edge node and 6 user buildings. It was also confirmed by the packet monitoring that the failure restoration function worked properly, when a failure was caused intentionally. It took 1-2 seconds to re-establish the IP connections and send the data automatially, which is smaller than the normal value of the time-out in IP-based applications. All the OTRs and L3SWs used in the experiment were the products in LAN for Ethernet use, including the routing protocols. This approach enables the network administrators in the CO-MAN to continue the administration and management jobs for the CO-MAN in the same way as those for the LAN inside the buildings.

The proposed network is scalable by adding/removing optical components and OTRs without any changes of installed optical fibers. In this experiment, the results are shown by using gigabit OTRs with 9 wavelengths of CWDM standard. Therefore, the maximum capacity is $9 \mathrm{Gbps}$ in the ring. More capacity can be transmitted, when using 10 Gbps OTRs, if more demands appear. Dense WDM can be used within the $20 \mathrm{~nm}$ spacing, if more wavelengths are necessary, where the same scheme of integrating 2 links can be adopted by assigning suitable wavelengths. This is the matter how applying the integration scheme proposed in this paper, and the verification is the further study.

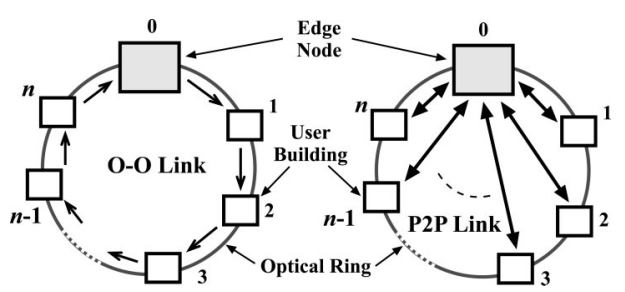

Fig. 1. Links in CO-MAN ring

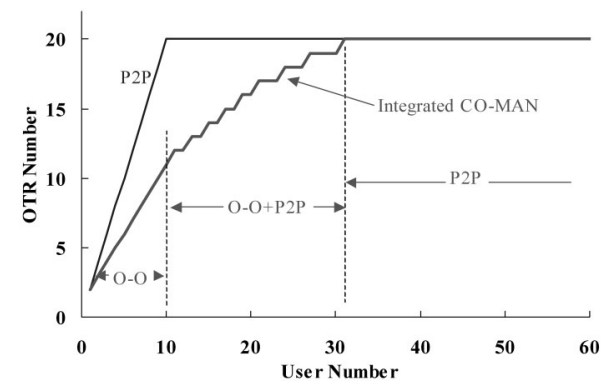

Fig. 2. Required OTR number

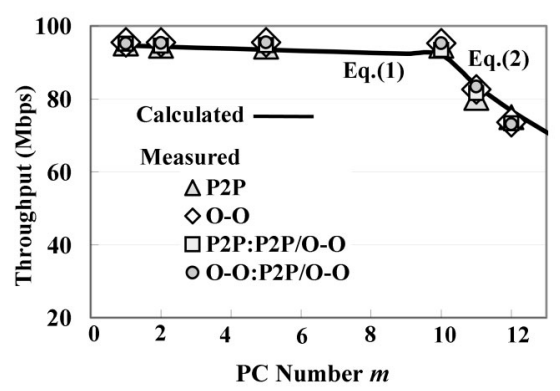

Fig. 3. Measured throughputs in the ring 


\title{
Integrated Native-Ethernet Optical Ring Networks for Scalable Customer-Owned Application
}

\author{
Satoru Fujimoto* \\ Taiji Sakamoto* \\ Non-member \\ Osanori Koyama* \\ Non-member \\ Yutaka Katsuyama* Member
}

\begin{abstract}
We have proposed and examined one-by-one (O-O) and point-to-point (P2P) link integration scheme over native-Ethernet optical ring networks for scalable customer-owned metropolican area network application (CO-MAN). The 2-link integration enables us to construct economical networks, which was clarified by smaller number of optical transceivers required to provide IP services. The experiments clarified that the integration of the $\mathrm{O}-\mathrm{O}$ and $\mathrm{P} 2 \mathrm{P}$ links was successfully made through $35 \mathrm{~km}$ long fiber ring. The failure restoraion function was also confirmed to work properly, when the service ring failure occurred. All the packet transmissions were made on the native-Ethernet base, which allows the network administrators of the CO-MAN to manage the network in the same way as in the commonly-used LANs.
\end{abstract}

Keywords: Optical MAN, IP-over-WDM, optical ring network, enterprise network

\section{Introduction}

Optical fiber networks are expected to satisfy the increasing traffic demands, by utilizing the high-capacity and low-loss characteristics. This trend is the same in WAN (wide area network) and MAN (metropolitan area network) applications to connect buildings/offices for business use, which are often referred to as intranet, or enterprise networks.

To transmit such business data, cell relay and frame relay services are used in public networks. Recently IP-VPN (Internet Protocol-Virtual Private Network) or wide Ethernet service is used more often than the cell and frame relay services, mainly due to the cost advantages $^{(1)}$. More IP and Ethernet transmissions are used, because many services are integrated and provided over IP, including business data storage and processing by web servers and VoIP (Voice-over-IP) service. This solution by the IP-VPN or wide Ethernet is based on the existing public networks, which are composed of high-speed SDH (Synchronous Digital Hierarchy) and ATM (Asynchronous Transfer Mode) networks. To transmit IP packets, high-speed routers to convert the IP/SDH/ATM transmissions are required as interfaces between the users and public networks in this solution.

Another solution is the dark-fiber or customer-owned networks ${ }^{(2)}$. This new type of network is becoming increasingly common among large enterprise networks, university research networks, and government departments in the USA and Canada. In the networks, en-

\footnotetext{
* The authors are with Graduate School of Engineering, Osaka Prefecture University, Gakuen-Cho 1-1, Sakai-shi, Osaka,

599-8531, Japan
}

terprises, government departments, and other organizations acquire optical fibers for their own communication services. The big advantage of the customer-owned networks is much lower cost for one-time capital cost for the fibers, instead of conventional cost per month for bandwidth. Thereafter, any increase in bandwidth only requires a simple equipment upgrade. It is another advantage that the networks can be managed and controlled, according to the organization policy.

Comparing the 2 solutions, the customer-owned networks give us an additional option to the enterprise networks, which provide a potentially low-cost approach, especially in large organizations. However, only very few papers were published, concerning network designs and performance. Contrary to this, many results were published ${ }^{(3)-(8)}$ for the public access and regional networks provided by common carriers.

Such technical background motivated us to investigate customer-owned MAN (CO-MAN) to provide IP services for their own use. The optical fibers in COMAN should be used effectively in a simple and skillless way, controlled by the network administrators. The requirements address the issues of the network scalability for the upgrade from low to high capacity of the transmission. Ethernet technologies are accepted and used widely in private LAN (local area network). This means that CO-MAN should be configured on the native Ethernet base for the easy management. The native Ethernet approach enables us to use existing transmission/routing equipments in a large LAN market, and not to use converters from/to IP/SDH/ATM, which must be used in public networks. Here, native Ethernet means the existing Ethernet technologies used in LAN so as to distinguish it from the modified Ethernet technologies 
with such converters for use in public networks.

The fundamental link on the native Ethernet base is the point-to-point (P2P) link, which is used commonly in LANs. The P2P link is also defined and standardized $^{(9)}$ in IEEE802.3 Task Force. Thus, P2P links can be used for CO-MAN. Though the P2P links are suitable to aggregate traffic from users to a central station, as in public networks, more traffic appears between buildings in CO-MAN. Therefore, different types of link are necessary, and should be integrated in CO-MAN in a simple and economical way. Thus, suitable network configurations should be clarified. We propose scalable optical IP networks owned by enterprises for their own use. The model networks followed by the proposed configurations were constructed, and the performance was examined. The design consideration and the performance are described in this paper.

\section{Integrated Scalable Networks for CO-MAN}

Generally the single star and ring topologies are used for business data transmission in WAN and MAN, due to the cost-effective transmission for large traffic, whereas PON (Passive Optical Network) topology is used for residential users to reduce the cost per user by sharing the central office equipment and optical fibers. Optical network configurations are classified ${ }^{(3)}$ as ring and tree topologies, when applying to WAN and MAN. Many of reported networks are the ring topology in the public networks, due to the scalability, reconfigurability and ease of protection. We also adopt the ring topology for the native Ethernet transmission over the scalable COMAN.

2.1 Proposal of 2-link Integration Scheme Fig. 1. shows a general configuration of CO-MAN considered in this paper. An edge node and user buildings (UBs) are connected with single-mode (SM) optical fibers to form a ring. The edge node and user interfaces (IFs) in the UBs have layer-3 electrical switches (L3SWs) or routers, optical transceivers (OTRs) and optical interface (IF), respectively. Wavelength-division multiplexing (WDM) technologies are used to have optical links through the ring. The optical IF denotes a component to mux/demux optical wavelengths for the WDM purpose, e.g. wavelength-sensitive optical coupler/splitter and ADM (add/drop multiplexer). The edge node aggregates packets from the UBs and send them to public networks through upper interface (U-IF), or send them to the UBs, depending on the destinations.

We propose CO-MAN configuration such that 2 optical links, shown in Fig. 2, are integrated in a ring: One is the one-by-one (O-O) link proposed in this paper. The O-O link is made by one wavelength, which is added/dropped by an ADM in each UB. The packets are routed by the O-O routing by the L3SW in the building. If the destination of the received packet is one of the personal computers (PCs) in the UB, the packet is sent to the PC. Otherwise, the packet is sent to the next UB. Thus, packets are circulated and checked oneby-one whether the final destination is the UB or not. The other one is the P2P links between the edge node

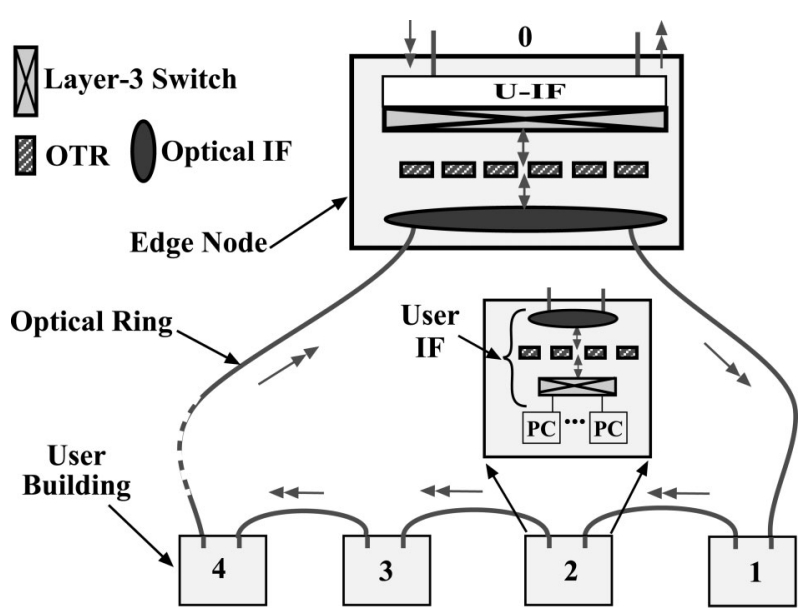

Fig. 1. General configuration of CO-MAN

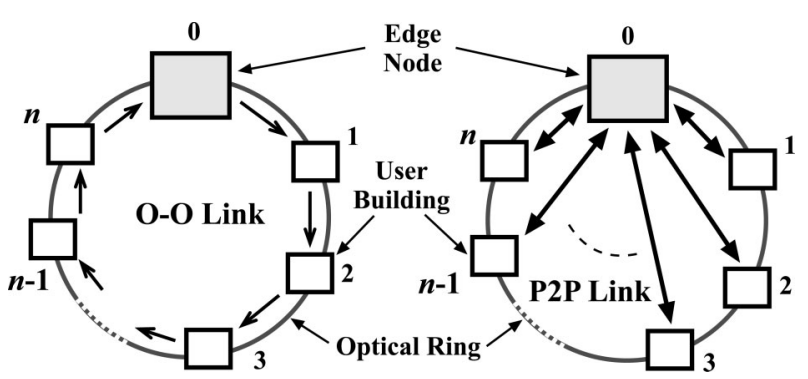

Fig. 2. Links in CO-MAN ring

and the UBs, which can be established in the optical ring by allocating one wavelength to each UB. The allocated wavelengths are multiplexed with a coupler in the edge node and one of the wavelengths is dropped/added with an ADM in the UB. The actual configuration is described in detail below.

To show the applicability of this network, a case study was made to design the network under particular conditions: The minimum OTR number was evaluated, required to provide services to a given number of users. Here, number of users means independent service number which should be provided inside the CO-MAN. The conditions were as follows: (1)1000Base-LX OTRs with different wavelengths are used in the edge node and UBs. (2)100Mbps should be guaranteed to each user in at most 10 different UB locations. (3) O-O link is adopted first to satisfy demands, and a P2P link is integrated, if the bandwidth of the O-O link is full. (4)Users are distributed uniformly among the 10 locations. In this case study, uniform user distribution was assumed to evaluate a costly case for a small number of users. When many users are concentrated in a location, the demands can be multiplexed, leading to an economical solution.

The minimum OTR number required to give optical links in the fiber ring can be determined as shown in Fig. 3. When UB number $n$ is equal to or less than 10 , one user exists in each UB, according to the condition (4), and one O-O link is enough to provide $100 \mathrm{Mbps}$. In case of one $\mathrm{UB}(n=1)$, the $\mathrm{O}-\mathrm{O}$ link is equal to $\mathrm{P} 2 \mathrm{P}$ link, and 2 OTRs (each in edge node and UB) are required. In case of 2 UBs $(n=2)$, one OTR is implemented in the 2nd UB location for the O-O link, and the 3 OTRs 


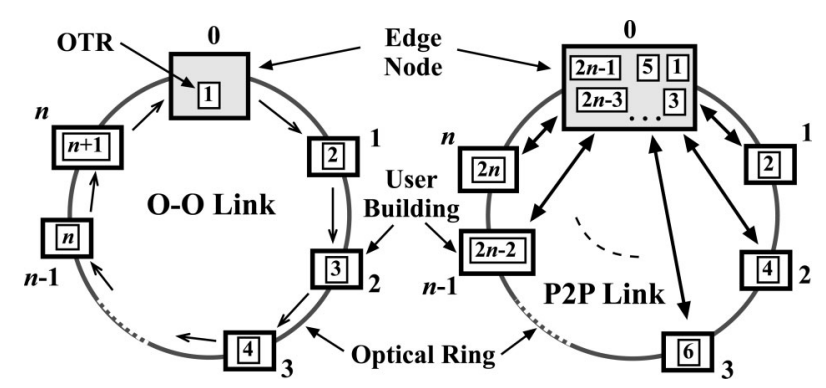

Fig. 3. Optical transceivers required in each link

are connected with fibers. Thus, OTR number for O-O link increases by $n+1$. Contrary to this, OTR number increases by $2 n$ to have P2P links, as shown in Fig. 3 .

The required OTR number is shown in Fig. 4. When user number is less than or equal to 10 , the required OTR number increases by $n+1$ for O-O link and by $2 n$ for P2P link, as described above. When user number is larger than 10, UB number remains 10, according to the condition (2), and multiple users exist in at least one UB. When user number is 11,2 users exist in one UB. The bandwidth provided by the O-O link is less than 100Mbps, and therefore, a P2P link should be added to the ring, according to the condition (3), leading to 2-OTR addition in the UB of the 2 users. The added P2P link can support the 2 user in the UB, and therefore, the OTR for the O-O link in the UB can be removed. As a result, the $\mathrm{O}-\mathrm{O}$ link is formed by connecting 9 UBs. When more users should be accommodated, and the bandwidth of the O-O link is full, one more $\mathrm{P} 2 \mathrm{P}$ link is added. In this way, OTRs are added until all the P2P links are added for the 10 UBs. For 31 users, 3 UBs contain users of 3,3 and 4 , respectively, and can be supported by one O-O link, and the other 7 UBs are supported by P2P links, respectively. This requires 18 OTRs. When user number is larger than 31, 20 OTRs for P2P links can provide 100Mbps for more than 32 to 100 users. Therefore, it is clear from Fig. 4 that the OTR number required to the integrated links is less than that required to the $\mathrm{P} 2 \mathrm{P}$ links. Thus, the proposed $\mathrm{O}-\mathrm{O}$ link and the integration scheme of $\mathrm{O}-\mathrm{O}$ and $\mathrm{P} 2 \mathrm{P}$ links for CO-MAN is the cost-effective solution.

In this case study, each $\mathrm{P} 2 \mathrm{P}$ link can satisfy the demands of 10 users in each UB, resulting in the accommodation of 100 users overall in 10 locations. When more demands than 100 users appear, the additional users can be accommodated in the same way as in Fig. 4. Thus, the integration of the 2 links has the cost advantage, depending on the user number.

2.2 Physical Network Configurations Fig. 5. shows an example of O-O link configuration, where each UB and the edge node have one OTR and one L3SW, respectively. Thus, this is the simplest configuration without any optical IFs, and is expected to be low cost to connect small number of users. The optical ring is formed by connecting a transmitting port of one OTR and a receiving port of the next OTR with a fiber in turn, as shown in Fig. 5. In this unique configuration, IP packets are circulated through the ring in one way,

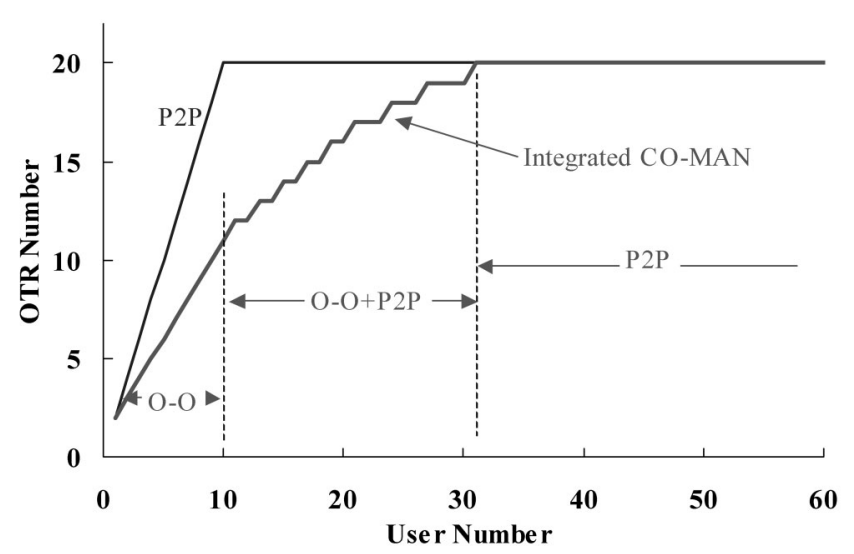

Fig. 4. Required OTR number

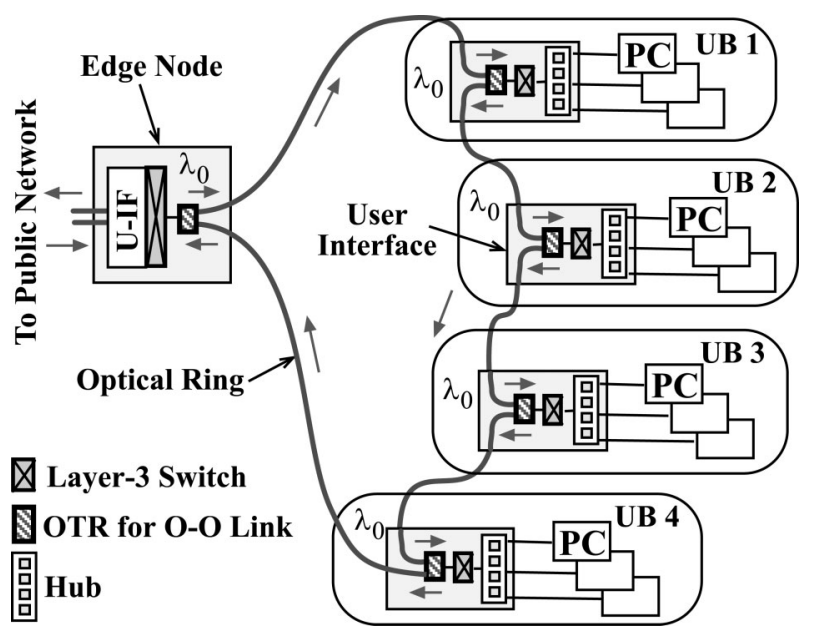

Fig. 5. O-O link configuration

contrary to the normal transmission between 2 OTRs in bi-directional way through 2 fibers. Thus, O-O link enables us to circulate packets through one fiber ring. The static IP routing is specified in the O-O link, which is described in section 3 , as well as the experimental results. The idea for the $\mathrm{O}-\mathrm{O}$ link is similar to that for the resilient packet ring ${ }^{(10)}$ (RPR). However, RPR was standardized to circulate RPR frames in a ring by a new method introduced in the MAC (Media Access Control) sub layer. Thus, RPR is not native Ethernet. The O-O link was proposed on a native Ethernet base for CO-MAN, where only the Ethernet technologies used in LAN were adopted for easy and skill-less management by administrators of the network owner.

An example of P2P link configuration is shown in Fig. 6. In this example, 4 wavelengths $\lambda_{1}-\lambda_{4}$ are used. One wavelength is allocated to one UB, where the allocated wavelength is added/dropped to/from the optical ring with the ADM. In the edge node, WDM coupler and splitter are used to mux/demux the wavelengths, and the packet routing is made by the L3SW, according to the destination addresses. Each UB has a direct link to the edge node with the allocated wavelength, though the physical topology of the optical fiber is a ring.

The O-O and P2P links can be integrated by adding a coupler and a splitter in the edge node in Fig. 5, and adding a $2 \lambda$-ADM or a $1 \lambda-\mathrm{ADM}$ in the $\mathrm{UB}$, as well as the 


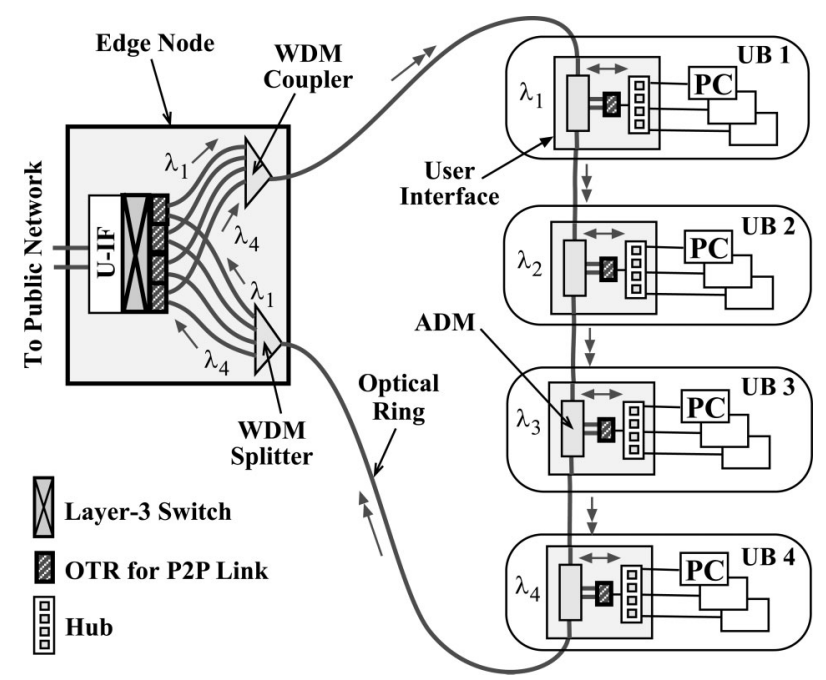

Fig. 6. P2P link configuration

OTRs. The $2 \lambda$-ADM has a function to add/drop a common wavelength $\lambda_{0}$ for O-O link and a particular wavelength $\lambda_{k}$ allocated to the UB $k$ for the P2P link, and $1 \lambda$-ADM adds/drops $\lambda_{0}$ for the O-O link. This optical IF and OTR addition process shows that the network is scalable without any changes of the installed fibers. The traffic demands can be satisfied from small to large user numbers in a cost-effective way by just adding/removing OTRs and optical IFs in the UBs and the edge node.

This is an example to show the upgrade by the 2-link integration. The network should be upgraded properly, according to the provisioning and demand estimation, in such a way that the upgrade costs are minimum. For example, more wavelengths than for the present small demands can be allocated and spare ADMs can be installed in advance, if it is clear that more demands appear in near future. It is possible to add one wavelength to a UB by connecting a $1 \lambda$-ADM to the existing ADM, but the adding works are not necessary, if a spare ADM has already been installed. This is the matter how the proposed 2-link integration scheme is applied to the actual upgrade, based on the demand estimation.

Figures 5 and 6 show 1-fiber ring with OTRs and optical IFs to provide IP services. Another fiber ring with same configuration of the OTRs and optical IFs should be added for the protection, when a failure occurs in the service ring. This additional ring is called a control ring in this paper. The overall configuration with the control ring is described in the next section. The control ring can be used as the bypass route, even if the L3SWs in the service ring are switched off, when OTRs and optical IFs are added/removed for the upgrade and reconfiguration processes. Thus, no practical degradation occurs in the service ring traffic, enabling us to keep the scalability of the network.

\section{Construction \& Evaluation of the Networks}

3.1 Experimental Network An integrated COMAN was constructed, and the performance was evaluated. Fig. 7 shows the experimental network configuration. The standard G.652 SM fibers were used to

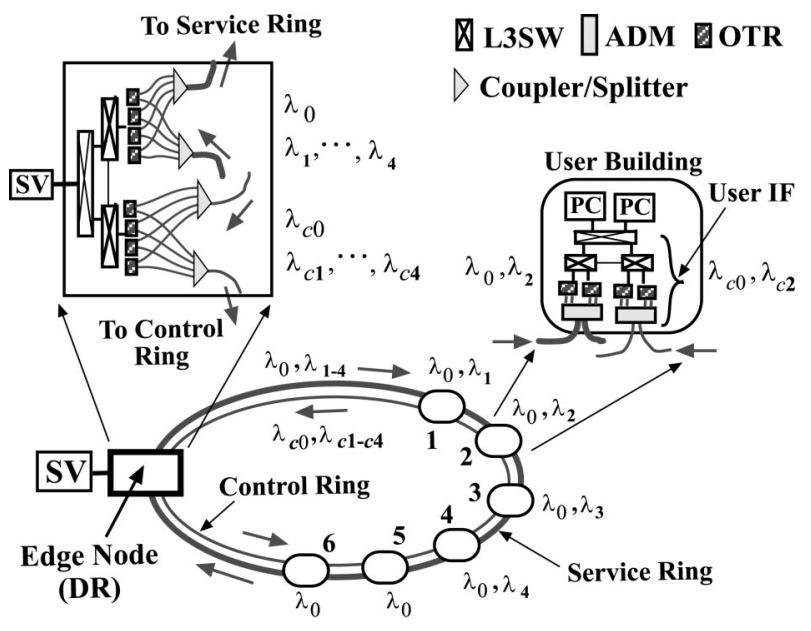

Fig. 7. Experimental network configuration

connect UBs. To integrate the optical links, an optical coupler and a splitter were fabricated to mux/demux 9 wavelengths of the coarse WDM (CWDM) standard: a wavelength of $\lambda_{0}(=1310 \mathrm{~nm})$ for O-O link, and 8 wavelengths from 1470 to $1610 \mathrm{~nm}$ with $20 \mathrm{~nm}$ spacing for P2P links were selected. Thus, the maximum number is 8 for the P2P links. The $2 \lambda$ - and $1 \lambda$-ADMs were used in the UBs. The $2 \lambda$-ADMs were constructed by connecting two $1 \lambda$-ADMs with particular wavelengths. The 8 wavelengths in 1550-nm band were allocated to the P2P links so as to have lower losses in optical fibers than 1310-nm band. This is because the lightwaves for the P2P links must pass ADMs, causing large losses in the $\mathrm{P} 2 \mathrm{P}$ links, as shown below.

Before evaluating the network shown in Fig. 7, the longest fiber length of the integrated service ring was evaluated. For the purpose, a service ring was constructed by connecting one edge node and $8 \mathrm{UBs}$, allocated one of the 8 wavelengths in the $1550 \mathrm{~nm}$-band to each UB. The standard 1000Base-LX OTRs with the 9 wavelengths were used for the tramsmission. Output powers of the OTRs were measured, and found to be more than $+2 \mathrm{dBm}$ and the minimum detectable level was $-20 \mathrm{dBm}$, leading to the dynamic range of $22 \mathrm{~dB}$. The losses of the optical IFs were measured. The loss for the downstream P2P links from the edge node to UBs is the total losses of the coupler, passing-through losses of the ADMs and dropping loss at the UB. The measured losses of the coupler/splitter ranged from 0.55 to $2.18 \mathrm{~dB}$, depending on the wavelengths. The measured passing-through and drop losses of the ADMs ranged from 0.7 to $1.66 \mathrm{~dB}$ and from 0.35 to $0.86 \mathrm{~dB}$, respectively. The largest loss for the downstream occurs for the farthest UB, which was measured and evaluated to be $9.21 \mathrm{~dB}$. The total loss and the dynamic range of OTRs give us the longest fiber length of $36.5 \mathrm{~km}$ for the downstream P2P link, assuming that the average losses of fibers and the splices are $0.35 \mathrm{~dB} / \mathrm{km}$ at $1550 \mathrm{~nm}$ band. In the same way, the longest fiber length was obtained for the upstream P2P link from the farthest UB to the edge node. The longest fiber length was also $36.5 \mathrm{~km}$.

The losses of the ADMs at wavelength $\lambda_{0}=1310 \mathrm{~nm}$ 
Table 1. Wavelengths allocated in the experiment

\begin{tabular}{|c|c|c|c|c|}
\hline Ring & \multicolumn{2}{|c|}{ Wavelength (nm) } & Link & UB \\
\hline \multirow{5}{*}{ Service } & $\overline{\lambda_{0}}$ & 1310 & $\mathrm{O}-\mathrm{O}$ & $1-6$ \\
\hline & $\lambda_{1}$ & 1470 & \multirow{4}{*}{$\mathrm{P} 2 \mathrm{P}$} & 1 \\
\hline & $\lambda_{2}$ & 1490 & & 2 \\
\hline & $\lambda_{3}$ & 1510 & & 3 \\
\hline & $\lambda_{4}$ & 1530 & & 4 \\
\hline \multirow{5}{*}{ Control } & $\lambda_{\mathrm{c} 0}$ & 1310 & $\mathrm{O}-\mathrm{O}$ & $1-6$ \\
\hline & $\lambda_{\mathrm{cl}}$ & 1470 & \multirow{4}{*}{$\mathrm{P} 2 \mathrm{P}$} & 1 \\
\hline & $\lambda_{\mathrm{c} 2}$ & 1490 & & 2 \\
\hline & $\lambda_{\mathrm{c} 3}$ & 1510 & & 3 \\
\hline & $\lambda_{\mathrm{c}}$ & 1530 & & 4 \\
\hline
\end{tabular}

were also measured to evaluate the O-O link. Since the $1310 \mathrm{~nm}$ lightwave is added/dropped at every UB, the optical losses are the summation of the adding and dropping losses of the ADM in the adjacent UBs. The total losses ranged from 0.24 to $0.79 \mathrm{~dB}$ for the O-O links. The link lengths of the segments between the adjacent UBs are more than $50 \mathrm{~km}$. As a result, it was clarified that the longest fiber length of optical ring can be 36.5 $\mathrm{km}$, satisfying both $\mathrm{P} 2 \mathrm{P}$ links at $1550 \mathrm{~nm}$-band and $\mathrm{O}-\mathrm{O}$ link at $1310 \mathrm{~nm}$ through one optical ring.

For the packet routing experiment, the optical ring connected an edge node and 6 UBs, as shown in Fig. 7. The fiber lengths between the edge node and UBs and between UBs were $5 \mathrm{~km}$, respectively. Therefore, total length of the ring was $35 \mathrm{~km}$. Four wavelengths from $\lambda_{1}$ to $\lambda_{4}$ were allocated to 4 of the $6 \mathrm{UBs}$ to give P2P links. Table 1 lists the wavelengths allocated to UBs in this experiment. Thus, $2 \lambda$-ADM was used in UBs 1-4, respectively, and $1 \lambda$-ADM was used in UBs 5 and 6 , respectively. A control ring was prepared for the service ring protection, as shown in Fig. 7. The same OTRs, optical IFs and L3SWs were installed in the control ring, and the L3SWs were connected in each UB, so as to make the traffic in the service ring be bypassed to the control ring, if service ring failure occurs. To have the interface between the L3SWs for the service and control rings and the outer traffic, an additional L3SW was installed and connected. The wavelengths used in the control ring are denoted by suffix $c$ in the symbols.

To send IP packets through the network, the routing functions should be implemented properly. We specified the dynamic routing for the $\mathrm{P} 2 \mathrm{P}$ links, and static routing for the $\mathrm{O}-\mathrm{O}$ links in the experimental network. Although OSPF (open shortest path first) is widely used protocol for the dynamic routing, it requires to have a designated router (DR) to which all other routers must be connected directly, according to the OSPF specification. It is possible for the $\mathrm{P} 2 \mathrm{P}$ links to be routed by OSPF, when the edge node is specified to be the DR. On the other hand, static routing was adopted in O-O links, because no DR could be specified. As a result, OSPF and static routing were configured in the L3SWs of UBs 1-4 to integrate P2P and O-O links, and only a static routing was configured in the L3SWs of UBs 5 and 6 .

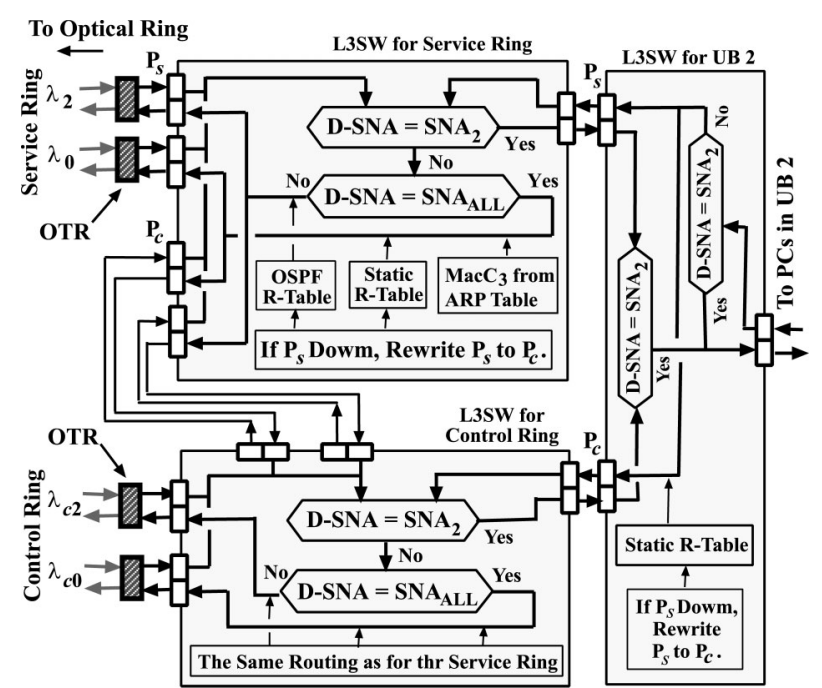

Fig. 8. Routing function implemented in UB 2

Fig. 8. shows the routing function implemented in the L3SWs of UB 2 to indicate an example of the P2P and $\mathrm{O}-\mathrm{O}$ link integration. In the L3SW for the service ring, the OSPF function was enabled so as to make a backbone area, made by the direct links to the edge node as DR. Therefore, the OSPF routing table (R-table) can be updated dynamically for all the P2P link states inside the ring. On the other hand, the routing entries for the O-O links were input statically and were saved in a static routing table. In this static routing table, all the sub network addresses of UBs connected in this ring were stored. The sub network addresses $\mathrm{SNA}_{1}, \mathrm{SNA}_{2}$, $\cdots, \mathrm{SNA}_{n}$ were denoted symbolically as SNA ALL in Fig. 8 , where $n$ is the UB number in this optical ring. Each $\mathrm{UB}$ was assigned different sub network address $\mathrm{SNA}_{k}$, where $k$ is a running number to denote one of the UBs.

When a packet is received by the L3SW for the service ring, the destination sub network address (D-SNA) is extracted from the destination IP address of the packet. The extracted D-SNA is compared with $\mathrm{SNA}_{2}$, as shown in Fig. 8. If the D-SNA and $\mathrm{SNA}_{2}$ are equal, then the packet is sent to the L3SW for UB2. This L3SW has a bypassing function for protection by giving a static routing similar to that in the L3SW for the service ring. When this L3SW receives a packet to UB2, the L3SW sends the packet to UB2 simply. The bypassing function for protection is described below. IF the D-SNA and $\mathrm{SNA}_{2}$ are not equal in the L3SW for the service ring, the D-SNA is compared with $\mathrm{SNA}_{\mathrm{ALL}}$. If the DSNA is equal to one of the SNA $\mathrm{ALL}$, the destination of the packet is inside the ring. The packet is sent to the port for the $\mathrm{O}-\mathrm{O}$ link, giving the MAC address $\mathrm{MacC}_{3}$ of the next L3SW in UB 3. The MAC addresses are stored in ARP (Address Resolution Protocol) table, which is input statically. The packet is converted to optical signal with a wavelength of $\lambda_{0}$. The D-SNA is not equal to anyone of the sub network addresses of SNA $\mathrm{ALL}$, then the packet is sent to the port for the P2P link. The packet is converted to optical signal with a wavelength of $\lambda_{2}$, leading to the direct link to the edge node for the 
transmission out of the ring.

In case of the service ring failure, the control ring is used. The failure restoration function was implemented in the L3SW for the service ring. As shown in Fig. 8, this function monitors all the ports $\mathrm{P}_{s}$ used for the service ring. If it is detected that $\mathrm{P}_{s}$ is down, then the routing table is rewritten in such a way that the output port is changed from $\mathrm{P}_{s}$ to $\mathrm{P}_{c}$, where $\mathrm{P}_{c}$ denotes the ports to send packets to the L3SW for the control ring. In the L3SW for the control ring, the packets to be sent to the ring are routed, in the same way as in the L3SW for the service ring. However, in the L3SW for the control ring, the packets to be sent to UB 2 are routed to the L3SW for UB 2, where the packets are sent simply to UB 2 . On the other hand, when users in UB 2 send packets, the packets are received by the L3SW for UB 2, where the packets are routed to the L3SW for the service ring. The packets are sent to the L3SW for the control ring, and then the packets are routed described above.

When a failure occurs in the L3SW for the service ring in UB 2, the two adjacent L3SWs for the service ring detect the link failures in the ports $\mathrm{P}_{s}$ in UB 1 and UB 3, respectively, because no communication is made due to the L3SW failure in UB 2. And the failure restoration functions rewrite the routing tables in UB 1 and UB 3 in the same way as in UB 2, respectively, so as to use the control ring. The processes in UB 1 and UB 3 cause the link failures in the L3SWs next to UB 1 and UB 3 , respectively. Thus, all the other L3SWs detect the link failure successively in the service ring, and rewrite the routing tables to use the control ring. On the other hand, packets sent by the users in UB 2 are received in the L3SW for UB 2, and are routed by the static routing table, as show in Fig. 8. The routing is specified to send packets to the port $\mathrm{P}_{s}$ in the normal transmission, when the destination is outside of UB 2. When it is detected that $\mathrm{P}_{s}$ is down in the L3SW for UB 2, the routing table is rewritten so as to use port $\mathrm{P}_{c}$. Thus, the control ring is used for the transmission of the packets sent from UB 2, when a failure occurs in the L3SW for the service ring.

In the same way, the routing function was implemented in the edge node. The routing configuration is shown in Fig. 9. The OSPF function was also enabled in this L3SW for the service ring, making it have a function of DR. When a packet is received by the L3SW for the service ring, the D-SNA is extracted and compared with sub network addresses $\left(\mathrm{SNA}_{\mathrm{ALL}}\right)$ of all the UBs in this ring, whose entries were updated dynamically by OSPF. If the compared result is "yes", then the output port is found by matching D-SNA with $\mathrm{SNA}_{k}$ of UB $k$, where $k=1,2,3$, or 4 in this experiment. And the packet is sent to the output port, leading to a conversion to the optical signal with a wavelength assigned to UB $k$. This optical signal is dropped by the ADM in UB $k$. Thus, direct P2P links can be made. If the extracted D-SNA is not equal to anyone of $\mathrm{SNA}_{k}(k=1,2,3$, or 4$)$, the packet destination is UB 5 or UB 6 , resulting in sending to port $\mathrm{P}_{s 0}$, which will be routed by the O-O link.

The failure restoration functions in the edge node were implemented in the same way as in UB 2. The monitoring function of the service ports $\mathrm{P}_{s k}$, and the rewriting function for the entries of the $\mathrm{P}_{s k}$ to the control ports $\mathrm{P}_{s i k}$ in the routing table were also implemented in the L3SW for the service ring for the failure restoration. The ports $\mathrm{P}_{s i k}$ of the L3SW for the service ring are connected to the ports $\mathrm{P}_{c i k}$ of the L3SW for the control ring. In case of the service ring failure, the control ring can be used through the paths from the ports $\mathrm{P}_{s i k}$ to $\mathrm{P}_{c i k}$, and the packets are routed by the L3SW for the control ring in the same way as in the L3SW for the service ring. When a failure occurs in the L3SW for the service ring, the L3SWs in UB 1 and UB 6 detect the link failures, respectively, and the failure restoration functions work so as to use the control ring in the same way as in the case of the L3SW failure in UB 2. The L3SW for UIF (Upper Interface) also detects the link failure in the L3SW for the service ring, and rewrite the routing table so as to use the L3SW for the control ring, in the same way as in the UB 2 .

3.2 Routing Characteristics of the Network The integrated CO-MAN ring was constructed, as shown in Fig. 7, and the routing functions were implemented, as shown in Figs. 8 and 9. The throughput was measured by sending packets from PCs in UBs to a server (SV) connected to the edge node, as shown in Fig. 7. The throughputs were obtained by measuring the transfer time, when the PCs sent the same size of data to SV simultaneously. The measurements were made with increasing PC number $m$, and the results are plotted in Fig. 10. Since the PCs had 100 Mbps network interface cards, respectively, $100 \mathrm{Mbps}$ data stream was added by increasing one PC. The measured results were almost the same value around $96 \mathrm{Mbps}$ when $m<11$, whereas it decreased when $m>10$. Theoretical throughput $t p(m)$

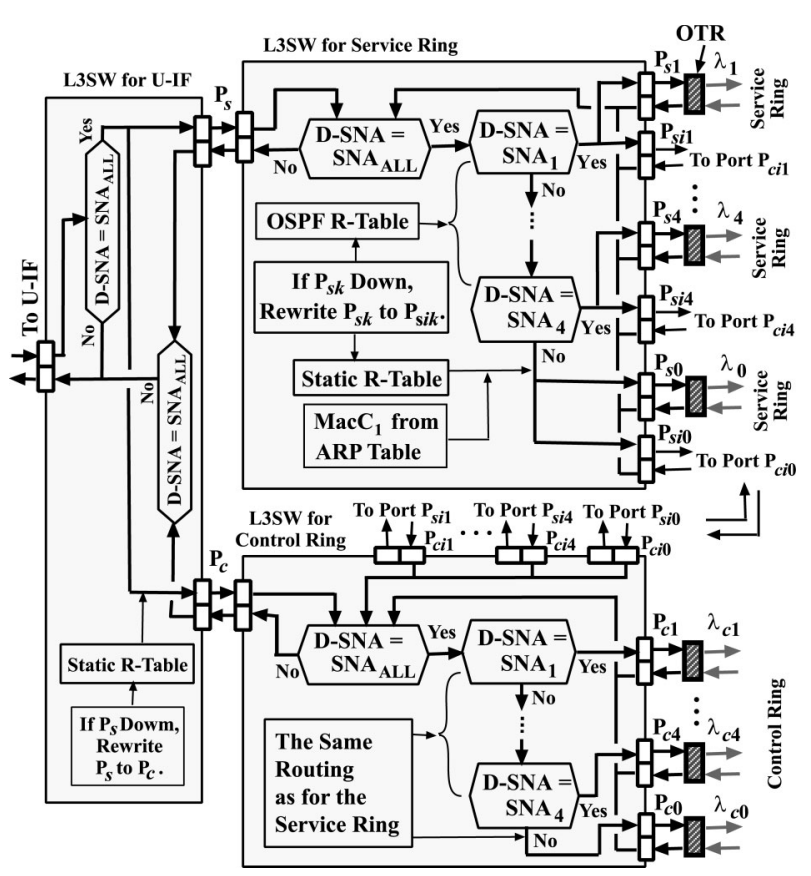

Fig. 9. Routing function implemented in the edge node 


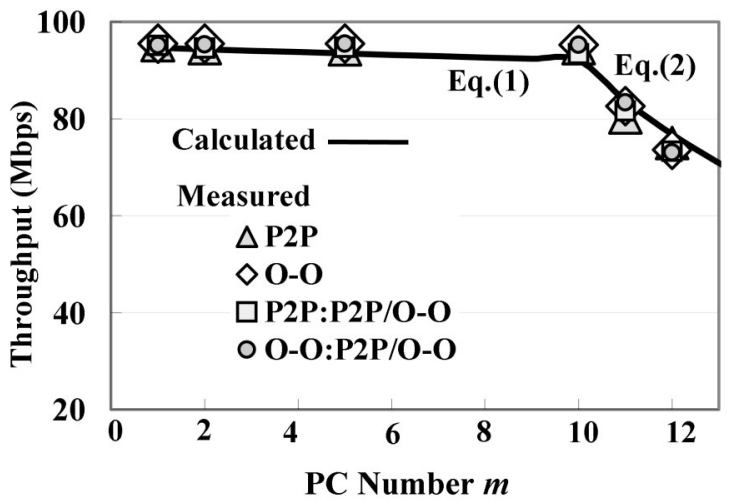

Fig. 10. Measured throughputs in the ring

$$
\begin{aligned}
& \text { is given }{ }^{(11)} \text { by } \\
& \begin{array}{ll}
t p(m)=u_{d} /\left(t_{u}+m z\right) & \left(m<m_{c r}+1\right) \cdots \\
t p(m)=u_{d} / m\left(t_{u}+z\right) & \left(m>m_{c r}\right) \cdots \cdots
\end{array}
\end{aligned}
$$

where $u_{d}, t_{u}$, and $z$ are unit packet size, unit transfer time which one packet $u_{d}$ is transferred, and a time which one packet passes through the L3SW, respectively. The value $u_{d}$ was 1460 byte in IEEE802.3 standard and the value $t_{u}$ was $0.122 \mathrm{~ms}$ in Eq. 1, which was given by 1526 bytes/100Mbps. Here, 1526 bytes are equal to the total bytes of $u_{d}$ and its header. In the same way, $t_{u}$ was $0.0122 \mathrm{~ms}$ in Eq. 2. The value $z$ was $0.001 \mathrm{~ms}$, which was obtained by 1526 bytes/9Gbps, because the inner bus speed was 9 Gbps in the L3SW. The parameter $m_{c r}$ is the critical value to represent the PC number by which all the bandwidth of 1 Gbps is exhausted by the $100 \mathrm{Mbps}$ streams sent by PCs, that is $m_{c r}=10$. The calculated curves are also shown in Fig. 10, indicating that the measured and calculated values are in good agreement. It is clear that the throughput was around $96 \mathrm{Mbps}$ for only $\mathrm{O}-\mathrm{O}$ and only $\mathrm{P} 2 \mathrm{P}$ links, respectively, when total traffic increased in the ring. The throughputs were also the same values, when $\mathrm{O}-\mathrm{O}$ and $\mathrm{P} 2 \mathrm{P}$ links were integrated. Thus, the integration was successfully made to provide nominal speed of $100 \mathrm{Mbps}$ to the users.

As the next experiment, the failure restoration functions were examined. While the traffic was transmitted simultaneously from each PC in all the UBs to the SV, the optical fiber in the service ring was disconnected intentionally to cause a failure. In the transmission, the packets were monitored with a packet analyzer: All the packets were monitored from the service and control ports in the L3SW of the edge node. The monitored results are shown in Fig. 11. Before the failure, the throughputs of the packets were smaller than about 950 Mbps. And after the failure, no essential transmission was made for a while, until small throughputs around $96 \mathrm{Mbps}$ appeared in the control ring. This shows that one of the connections was re-established through the control ring, and the transmission continued to be completed. After this transmission, the same throughputs around $96 \mathrm{Mbps}$ appeared, corresponding to one of the other traffic. And afterward, similar behavior was observed to the first transmission in the service ring before

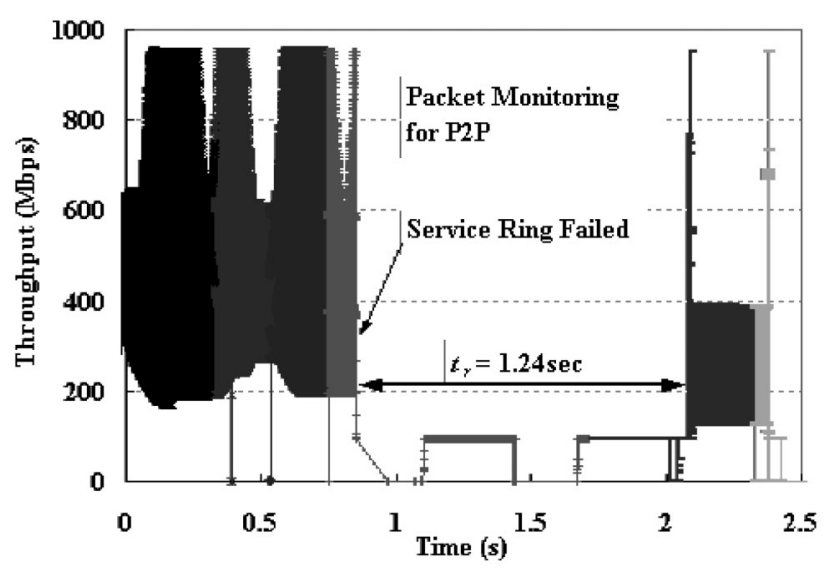

Fig. 11. Packet monitoring for P2P links

the failure, showing that the traffic for the remaining 2 links. In the processes, it is understood that the failure made the transmission stop in the service ring, activated the restoration function, and restarted the transmission, after the connections were re-established in the control ring. The overall time $t_{r}$ to re-establish all the connections was $t_{r}=1.24 \mathrm{~s}$. Thus, it is clear that the failure restoration function worked properly in the routing functions implemented in this configuration. Before and after the failure restoration process, the throughputs were smaller than about $950 \mathrm{Mbps}$, which were caused by the nominal speed difference of the $100-\mathrm{Mbps} \mathrm{PC}$ and 1Gbps OTR interfaces. The similar results were obtained for the monitoring of the $\mathrm{O}-\mathrm{O}$ link packets. The restoration time $t_{r}$ for the O-O link was around 2 seconds. The failure restoration functions were also examined, when a failure of the L3SW for the service ring occurred in the edge node. The failure was caused by switching the L3SW off. The values of measured restoration time $t_{r}$ were almost the same as in the service ring failures, respectively.

\section{Conclusion}

We have proposed and examined $\mathrm{O}-\mathrm{O}$ and $\mathrm{P} 2 \mathrm{P}$ link integration scheme over native-Ethernet optical ring networks for scalable customer-owned MAN application. The network configurations are described to integrate the 2 optical links by using CWDM technologies. The 2-link integration enables us to construct economical optical ring networks, which was clarified by smaller number of optical transceivers required to provide IP services. This is the essential econimical point, because the transmssion equipment costs are main, after the optical fibers are owned in the CO-MAN.

An experimental network was constructed on the native-Ethernet base and the performance was examined to clarify the integrated CO-MAN characteristics. An optical coupler and a splitter to mux/demux 9 wavelengths of CWDM standard were fabricated for use in an edge node, and $2 \lambda$ - and $1 \lambda$-ADMs were fabricated for use in user building. It was estimated from the measured losses and the dynamic range of 1000BaseLX transceivers used in the experiment that the optical 
fiber length of the ring could be longer than $36.5 \mathrm{~km}$ to integrate $8 \mathrm{P} 2 \mathrm{P}$ links and one $\mathrm{O}-\mathrm{O}$ link without optical amplification.

The packet routing functions were implemented in the L3SWs. The routing by P2P link was based on OSPF and that by $\mathrm{O}-\mathrm{O}$ link was based on static routing, including the packet bypassing function to the control ring, when a failure occurred in the service ring. The throughput measurement clarified that the integration of the $\mathrm{O}-\mathrm{O}$ and $\mathrm{P} 2 \mathrm{P}$ links was successfully made for the ring network connecting one edge node and 6 user buildings. It was also confirmed by the packet monitoring that the failure restoration function worked properly, when a failure was caused intentionally. It took 1-2 seconds to re-establish the IP connections and send the data automatially, which is smaller than the normal value of the time-out in IP-based applications. All the OTRs and L3SWs used in the experiment were the products in LAN for Ethernet use, including the routing protocols. This approach enables the network administrators in the CO-MAN to continue the administration and management jobs for the CO-MAN in the same way as those for the LAN inside the buildings.

The proposed network is scalable by adding/removing optical IFs and OTRs, when optical fibers are installed. In the experiment, the results are shown by using gigabit OTRs with 9 wavelengths of CWDM standard. Therefore, the maximum capacity is 9 Gbps in the ring. More capacity can be transmitted, when using 10 Gbps OTRs, if more demands appear. Dense WDM can be used within the $20 \mathrm{~nm}$ spacing, if more wavelengths are necessary, where the same scheme of integrating 2 links can be adopted by assigning suitable wavelengths. This is the matter how applying the integration scheme proposed in this paper, and the verification is the further study. The 2-link integration scheme was proposed, based on the case study for the unifor user distribution. The other scheme, including the integration of only O-O links, can be possible for different user distribution, which is also the further study.

\section{Acknowledgment}

The authors would like to thank students of the multimedia network research laboratory in Osaka Prefecture University for the experimental assistance.

(Manuscript received May 9, 2005,

revised Sep. 16, 2005)

\section{References}

(1) Nikkei: "Nikkei Communications", No.397, pp.84-87, (in Japanese)(2003)

(2) Bill St. Arnaud, Jing Wu, and Bahman Kalali: "Customercontrolled and -managed optical networks", J. Lightwave Tech., Vol.21, No.11, pp.2804-2810 (2003)

(3) Adel A. M. Saleh and Jane M. Simmons: "Architectural principles of optical regional and metropolitan access networks", J. Lightwave Tech., Vol.17, No.12, pp.2431-2448 (1999)

(4) W. T. Anderson, J. Jackel, G. -K. Chang, Dai Hongxing, Wei Xin, M. Goodman, C. Allyn, M. Alvarez, O. Clarke, A. Gottlieb, and F. Kleytman: "The MONET project-a final report", J. Lightwave Tech., Vol.18, No.12, pp.1988-2009 (2000)

(5) L. Berthelon, O. Audouin, P. Bonno, C. Drion, E. Grard, O.
Rofidal, C. M. Martin, E. Ringoot, I. van de Voorde, G. J. Eilenberger, and F. Raeymaekers: "Design of a cross-border optical core and access networking field trial: first outcomes of the ACTS-PELICAN project", J. Lightwave Tech., Vol.18, No.12, pp.1939-1954 (2000)

(6) N. Madamopoulos, D. Clint Friedman, I. Tomkos, and A. Boskovic: "Study of the performance of a transparent and reconfigurable metropolitan area network", J. Lightwave Tech., Vol.20, No.6, pp.937-945 (2002)

( 7 ) T. Aihara, M. Kawakami, Y. Enomoto, T. Tashiro, and K. Sato: "Ring network architecture using wavelength division multiplexing coupler for metropolitan area networks", Proc. of OECC2002, pp.562-563, Yokohama, Japan, Jul.(2002)

( 8 ) K. Noguchi, Y. Koike, H. Tanobe, K. Harada, and M. Matsuoka: "Field trial of full-mesh WDM network (AWG-STAR) in metropolitan/local area", J. Lightwave Tech., Vol.22, No.2, pp.329-336 (2004)

(9) IEEE: Media Access Control Parameters, Physical Layers, and Management Parameters for Subscriber Access Networks, IEEE Std 802.3ah, (2004)

(10) IEEE: Resilient Packet Ring (RPR) Access Method and Physical Layer Specifications, IEEE Std 802.17, (2004)

(11) S. Fujimoto, K. Tamura, O. Koyama, and Y. Katsuyama: "High-speed transmission evaluation of gigabit local area networks by limiting performance model", Proc. of APCC, pp.335-338, Bandung, Indonesia, (2002)

Satoru Fujimoto (Non-member) was born in 1978. He re-

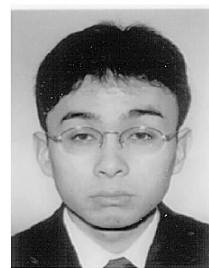
ceived the B.S. and M.S. degrees in Electrical \& Electronic Systems from Osaka Prefecture University in 2001 and 2003, respectively. Since 2003, he has been a student in the doctor course in the Graduate School of Engineering, Osaka Prefecture University, Japan. His research interests include optical IP network design and evaluation.

Taiji Sakamoto (Non-member) received the B.S. in Electri-

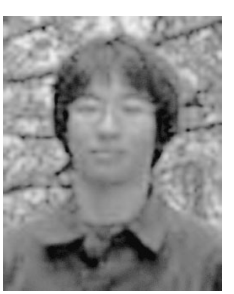
cal \& Electronic Systems from Osaka Prefecture University in 2004. Now he is a master course student in Osaka Prefecture University, Japan. His research interests include optical access network design.

Osanori Koyama (Non-member) was born in 1976. He re-

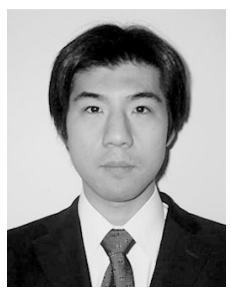
ceived the B.S. and M.S. degrees in Electrical \& Electronic Systems from Osaka Prefecture University in 1999 and 2001, respectively. Since 2001, he has been a research assistant in the Graduate School of Engineering, Osaka Prefecture University, Japan. His research interests include design and control aspects of IP networks.

Yutaka Katsuyama (Member) was born in 1949. He re-

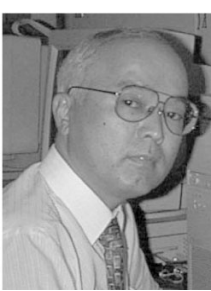
ceived the B.S., M.S. and PhD degrees in optics \& communication field, all from Kyoto University in 1971, 1973 and 1981, respectively. Since 1973, he was a research engineer in NTT Electrical Communications Laboratory till 1997, where his major work was research and developmental works on optical fiber cable design and evaluation. Since 1997 he has been a professor in the Graduate School of Engineering, Osaka Prefecture University, Japan. His research interests include design and evaluation of optical IP networks. 